Copyright 2012 American Institute of Physics. This article may be downloaded for personal use only. Any other use requires prior permission of the author and the American Institute of Physics. The following article appeared in Journal of Applied Physics 111(7) and may be found at: DOI: $10.1063 / 1.3688933$.

\title{
Concentration Dependence of Magnetic Moment in $\mathrm{Ce}_{1-\mathrm{x}} \mathrm{Fe}_{\mathrm{x}} \mathrm{O}_{2}$
}

\author{
Geoffrey L. Beausoleil II, Aaron Thurber, S.S. Rao, Gordon Alanko, Charles B. Hanna, and Alex Punnoose \\ Boise State University
}

\begin{abstract}
In this study, we examined the impact of iron doping on the structural, chemical, and magnetic properties of ceria $\left(\mathrm{Ce}_{1-\mathrm{x}} \mathrm{Fe}_{\mathrm{x}} \mathrm{O}_{2}\right)$. Samples were produced in triplicate through a coprecipitation approach in a forced hydrolysis synthesis that yielded consistently sized nanocrystals, using three different cerium precursors: cerium chloride, cerium ammonium nitrate and cerium nitrate. Particles were characterized by x-ray diffraction (XRD), x-ray photoelectron spectroscopy (XPS), transmission electron microscopy (TEM), electron paramagnetic resonance (EPR) spectroscopy and vibrating sample magnetometry (VSM). XRD showed the average crystallite size to be $4.4 \pm 1.2 \mathrm{~nm}$, which agreed well with TEM data. XPS and EPR data shows iron to be in the $\mathrm{Fe}^{3+}$ state and confirms the nominal dopant concentration. The moment per $\mathrm{Fe}$ ion found was largest at the lowest dopant concentrations, with values of 0.078 and $0.055 \mu_{\mathrm{B}} / \mathrm{Fe}$ ion for 0.1 and $0.5 \% \mathrm{Fe}$, respectively, which quickly decreased as the concentration was increased. We used XPS to estimate $\mathrm{Ce}^{3+} / \mathrm{Ce}^{4+}$ ratio to be in the 0.2-0.3 range and observed a linear relation between the saturation magnetization and the $\mathrm{Ce}^{3+} / \mathrm{Ce}^{4+}$ ratio.
\end{abstract}

\section{Introduction:}

Extensive research has been done over the last decade exploring transition metal (TM) doping of metal oxide nanoparticles (NPs) for potential use in spintronic devices. It has been suggested by Sundaresan et al. $(1,2)$ that room-temperature ferromagnetism (RTFM) is exhibited universally by inorganic NPs due to exchange between electron spin moments caused by oxygen vacancies $\left(\mathrm{V}_{\mathrm{O}}\right)$. Ge et al. (3) theorized and verified in experiment that $\mathrm{V}_{\mathrm{O}}$ causes a significant increase in magnetization of NPs over bulk samples, and that $\mathrm{V}_{\mathrm{O}}$ affects the surrounding $f$ electrons , causing a net magnetic moment for the particles. It was also suggested that the $\mathrm{V}_{\mathrm{O}}$ concentration can be increased by annealing in a reducing atmosphere $(4,5)$, and Li et al. (4) showed that switching annealing gases from oxidizing to reducing atmospheres will alter the magnetization accordingly.

In addition to oxygen vacancies, TM doping has been shown to increase the RTFM of ceria NPs (6-13). There are at least two possible mechanisms for RTFM enhancement by TM doping: creation of oxygen vacancies by introduction of a different-valance dopant into the host lattice and ferromagnetic ordering of a TM dopant. The contribution of iron to RTFM of nanoparticles is state-dependent. $\mathrm{Fe}^{2+}$ contributes no unpaired electrons to ferromagnetic ordering, while $\mathrm{Fe}^{3+}$ ions can contribute to ferromagnetic ordering. For these reasons, we synthesized Fe-doped $\mathrm{CeO}_{2}$ in the same reaction system, using 3 different precursors in an effort to produce variation in the Fe valence state and $V_{O}$ concentration, in order to examine the effect of both parameters on RTFM in $\mathrm{CeO}_{2} \mathrm{NPs}$.

\section{Experiment:}

Ceria NPs were prepared by a forced-hydrolysis process using three different cerium precursors: Cerium chloride (Ce-Cl), Cerium ammonium Nitrate (Ce-AmNit), and Cerium nitrate (Ce-Nit). The cerium precursor was dissolved along with Lithium hydroxide in ethanol, heated to $70^{\circ} \mathrm{C}$ in a silicon oil bath, and held while stirring for 90 minutes. After heating, the solution was mixed with N-heptane to facilitate crystal growth, and allowed to rest for 20-24 hours. Then the precipitate was centrifuged out and washed in ethanol to remove any remaining precursor, and twice washed in nano-pure water to remove any residual hydroxide and ethanol. The final product was dried in an oven for 24 hours at $50^{\circ} \mathrm{C}$ before being ground to a fine powder using an agate mortar and pestle. Iron-doped samples were prepared by the same method, substituting a calculated molar ratio of Iron chloride (in $\mathrm{Ce}-\mathrm{Cl}$ ) or Iron nitrate (in Ce-Nit and Ce-AmNit) for the cerium precursor to form $\mathrm{Ce}_{1-\mathrm{x}} \mathrm{Fe}_{\mathrm{x}} \mathrm{O}_{2-\delta}$. In the Ce-Cl system, $\mathrm{x}=0.001$, 0.005, 0.01, 0.025, 0.05, 0.075, 0.10, 0.15 and 0.20. In the Ce-Nit and Ce-AmNit systems, $x=0.01,0.05$, and 0.10 were used to verify trends against $\mathrm{Ce}-\mathrm{Cl}$. All samples were made in triplicate to show consistency and reproducibility in the results. 
Copyright 2012 American Institute of Physics. This article may be downloaded for personal use only. Any other use requires prior permission of the author and the American Institute of Physics. The following article appeared in Journal of Applied Physics 111(7) and may be found at: DOI: $10.1063 / 1.3688933$.

Characterization of samples was done as soon as possible after synthesis to avoid possible deformation or phase change of the samples. X-ray diffraction (XRD) was done on a Phillips X'Pert X-ray diffractometer using BraggBrentano geometry with a $\mathrm{Cu} K_{\alpha}$ source with $\lambda=1.5418 \AA$, with particle size determined by the Scherrer equation and lattice parameters calculated from the Bragg equation. Magnetic measurements were done on a Lake Shore 7404 Vibrating Sample Magnetometer (VSM) at room temperature and ambient conditions, on a range of $\pm 10 \mathrm{kG}$. Dopant spin state and magnetic behavior were investigated using a conventional first derivative X-band $(\sim 9.392$ $\mathrm{GHz}$ ) electron paramagnetic resonance (EPR) experiment at $\mathrm{T}=5 \mathrm{~K}$ and $\mathrm{T}=295 \mathrm{~K}$, using a Bruker ELEXYS EPR spectrometer equipped with continuous flow Oxford cryostat. X-ray Photoelectron spectroscopy (XPS) was carried out on a PHI 5000 VersaProbe XPS with a Al- $\mathrm{K}_{\alpha} \mathrm{X}$-ray. The elemental concentration was determined for each sample by curve fitting the XPS data using the software programs available with the XPS unit TEM images were taken on a Joel JEM-2100 HR TEM.

\section{Results and Discussion:}

XRD patterns for both pure and doped samples showed the typical $\mathrm{CeO}_{2}$ fluorite cubic structure, with no indication of other cerium or iron phases (see Fig 1). Using an average of multiple peaks fitted using a Lorentzian peak fit, particle size and lattice parameter were found. Pure ceria NPs made with Ce-Cl had an average size of $4.4 \mathrm{~nm} \pm 1.24$

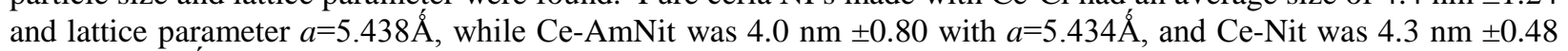
with $a=5.431 \AA$. Size distributions calculated from TEM images agreed with that found in XRD. SAED patterns had d-spacing consistent with the cubic fluorite structure.

XRD was also used to determine whether Fe dopant ions were interstitial or substitutional in the $\mathrm{CeO}_{2}$ lattice. It is expected that substitutional doping of $\mathrm{Fe}^{3+}$ (ionic radius 55pm) for $\mathrm{Ce}^{4+}$ (ionic radius $114 \mathrm{pm}$ ) would decrease the lattice spacing with increasing doping, while interstitial doping would cause the lattice parameter to expand with increasing doping. This is important, because it is predicted that substitutional doping of different-valence TM atoms introduces oxygen vacancies, helping to facilitate stronger RTFM from unpaired electron interactions. In the case of iron doping in these samples, Fig 2 shows that in all three sample groups, there was a sharp dip in the lattice parameter at lower iron concentrations $(x<2.5 \%)$, indicating substitutional doping. For higher concentrations (2.5$20 \%$ ), the lattice parameter decreased, but at a much slower rate, which could indicate a combination of substitutional and interstitial doping.

XPS was done on the samples to determine $\mathrm{Ce}^{3+/ 4+}$ ratios and the valence state of iron in the doped samples, as shown in Fig 3. Ce 3d spectra were simultaneously fited with Gauss-Lorentz curves representing both $\mathrm{Ce}^{4+}$ and $\mathrm{Ce}^{3+}$ as well as their shake-up satellites, and the ratio $\mathrm{Ce}^{3+} / \mathrm{Ce}^{4+}$ was calculated from the main fitted peaks. It was found that the Ce-Cl had a Ce ${ }^{3+/ 4+}$ ratio close to 0.3 , the Ce-Nit close to 0.25 , and the Ce-AmNit close to 0.2. The XPS spectra of the Fe 3p peak region of the Fe doped ceria samples are shown in Figs. 3B and 3C respectively, with drop lines indicating the reported positions of the $\mathrm{Fe}^{3+}$ and $\mathrm{Fe}^{2+}$ peaks (14). The positions of peaks for all of the doped samples, and the lack of shoulders on the peaks, indicate that the iron present is in the 3+ state. Also shown in Fig. 3A is a simultaneous Gauss-Lorentz curve fit for the 3p peak with components at 55.4 and $55.85 \mathrm{eV}$, both of which match literature values for $\mathrm{Fe}^{3+}$ (14). There is little evidence for the presence of $\mathrm{Fe}^{2+}$ in the iron doped ceria samples prepared using any of the 3 methods, and with the steady growth in signal strength from the $\mathrm{Fe}^{3+}$ peak, we can expect to see an increasing ferromagnetic signal from the samples as a result (7). The Ce-AmNit system, shown in Fig 3B, shows a slight shift in the Fe 3p peak toward lower binding energy, which may indicate a higher fraction of $\mathrm{Fe}$ present as $\mathrm{Fe}^{2+}$. In order to confirm the oxidation state of Fe ions further, EPR measurements were performed on all the Fe doped samples at $5 \mathrm{~K}$. Fig. 4 shows representative spectra from two selected samples, which are very similar to that reported for $\mathrm{Fe}^{3+}$ ions in similar systems (15), thus further confirming the 3+ state of our Fe dopants.

VSM measurements showed very weak ferromagnetic signals from pure ceria samples in all three methods. The saturation magnetization $\mathrm{M}_{\mathrm{s}}$ of the pure samples was $4.03 \times 10^{-4} \mathrm{emu} / \mathrm{g}$ for Ce-Cl, $4.23 \times 10^{-4} \mathrm{emu} / \mathrm{g}$ for Ce-Nit, and $1.35 \times 10^{-4} \mathrm{emu} / \mathrm{g}$ for Ce-AmNit. The coercive force for these was 30-115 G for Ce-Cl and 25-110 G for Ce-Nit. The Ce-AmNit samples did not have a measurable coercivity. For iron doping, Ce-Cl samples with a broad variation in iron percentage were made and measured, while for Ce-Nit and Ce-AmNit samples only a few percentages were made for comparison. The iron doped Ce-Cl samples, shown in Fig. 5A, exhibit a steady increase in magnetization as the percentage of iron doping increases, which matches the previously stated expectation of finding a higher magnetic signal due to increased concentration of $\mathrm{Fe}^{3+}$. The Ce-Nit samples also show a similar 
Copyright 2012 American Institute of Physics. This article may be downloaded for personal use only. Any other use requires prior permission of the author and the American Institute of Physics. The following article appeared in Journal of Applied Physics 111(7) and may be found at: DOI: $10.1063 / 1.3688933$.

pattern: increasing magnetism with increasing iron percentage, presumably due to the formation of $\mathrm{V}_{\mathrm{O}}$ and/or the interaction between the free electrons of the $\mathrm{Fe}^{3+}(3)$.

Saturation magnetization $\mathrm{M}_{\mathrm{s}}$ of the iron doped Ce-AmNit samples did not show the same pattern as the Ce-Cl or CeNit, but instead remained unaffected by the increase in iron concentration (see Fig. 5C). It was determined through XPS measurements that the $\mathrm{Ce}^{3+/ 4+}$ ratios in the Ce-AmNit samples were the lowest (Fig. 6) and $\mathrm{M}_{\mathrm{s}}$ of the Fe doped samples, in general, increases linearly with $\mathrm{Ce}^{3+/ 4+}$ ratios. Fig. 6 would suggest that there is a lower concentration of $\mathrm{V}_{\mathrm{O}}$ caused by cerium reduction in the Ce-AmNit samples. A key difference between the Ce-AmNit samples and the others is that the Fe 3p peaks are slightly shifted to a lower binding energy that better matches to the positions of the $\mathrm{Fe}^{2+}$, see Fig. 3B. However, in order to maintain charge neutrality, oxygen vacancies might be formed as a result of $\mathrm{Fe}^{2+}$ doping which, if vacancies were responsible for RTFM, would give rise to a noticeable increase in RTFM. Since it does not, we are left with the conclusion the RTFM is not being caused by oxygen vacancies, but rather free-electron interactions provided by $\mathrm{Fe}^{3+}$ ions. Another consideration is that any $\mathrm{Fe}^{3+}$ ions that are present may have their free electrons trapped in the oxygen vacancies created by $\mathrm{Fe}^{2+}$, as described by Dohčević et al. (7). Combining this with the results for $\mathrm{Ce}-\mathrm{Cl}$ and $\mathrm{Ce}-\mathrm{Nit}$ ions, we can say that the increased presence of $\mathrm{Fe}^{3+}$ ions contribute to an increase in RTFM for ceria NPs, and that there is little contribution from $\mathrm{V}_{\mathrm{O}}$.

\section{Conclusion:}

Three different sets of $\mathrm{Ce}_{1-\mathrm{x}} \mathrm{Fe}_{\mathrm{x}} \mathrm{O}_{2-\delta} \mathrm{NPs}$ were successfully created for comparing magnetic properties as a function of $\mathrm{Fe}$ and $\mathrm{V}_{\mathrm{O}}$. XRD analysis confirmed that the fluorite cubic structure of the samples was free of other phases, and that iron was successfully incorporated into the ceria structure. We did not find support for a theory of magnetic moment enhancement with increasing $\mathrm{V}_{\mathrm{O}}$, though further research could be done by doping nonmagnetic TM ions into the ceria lattice to provide further confirmation of this result. Through XPS and VSM analysis, it was seen that for Ce-Cl and Ce-Nit, the ferromagnetic ordering was enhanced with increasing Fe doping, due to interaction of free electrons from $\mathrm{Fe}^{3+}$ ions. The same inverse relationship for the magnetic moment was also found for the Ce-AmNit samples, but the magnetization of these samples was very low proabably due to the trapping of any unpaired electrons within $\mathrm{V}_{\mathrm{O}}$.

\section{Acknowledgements}

This work was supported in part by the National Science Foundation's EAGER grant (DMR-1137419), Grant No. W911NF-09-1-0051 from the Army Research Office, and the CBET grant 1134468 from the National Science Foundation. CBH was supported by NSF grant DMR-0906618. 

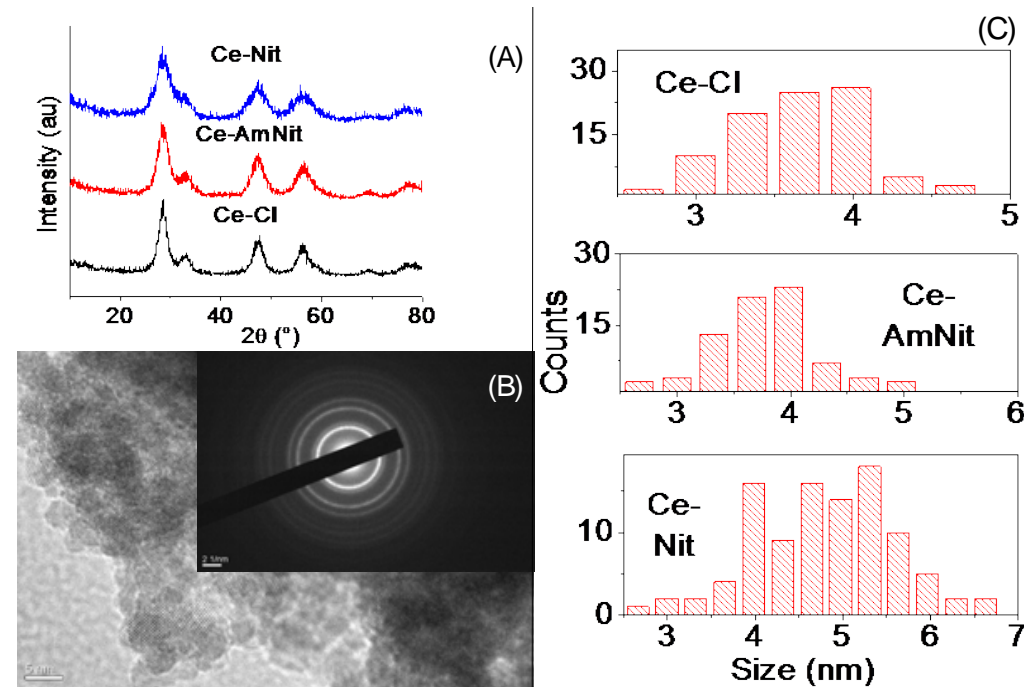

(C)

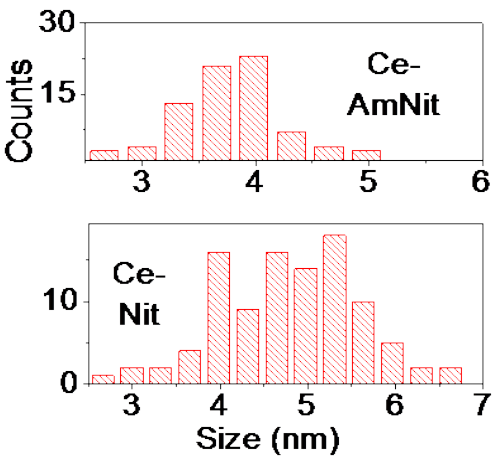

Figure 1 (color online) (A) XRD patterns for pure ceria samples. Through the Scherrer relation, the average size of the particles was determined to be about $4 \mathrm{~nm}$ for all three samples. (B-C) TEM image and diffraction pattern with size distribution.

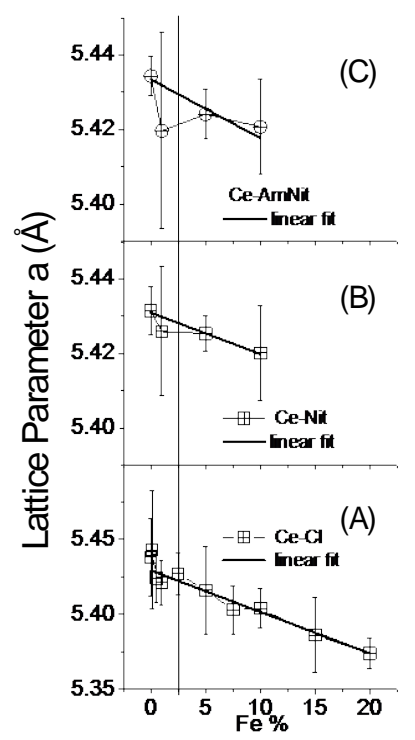

Figure 2 A-C) show the change in lattice parameters for Fe doped samples of Ce-Cl, Ce-Nit, and Ce-AmNit methods, respectively, with a linear fit applied. All three methods showed a strong "dip" in the lattice parameter for $\mathrm{x}<2.5 \%$, indicated by a drop line at $2.5 \%$. For Ce-Cl prepared samples, there was a more stable linear decline in the lattice parameter. This trend also appears to be present in the other two methods. 


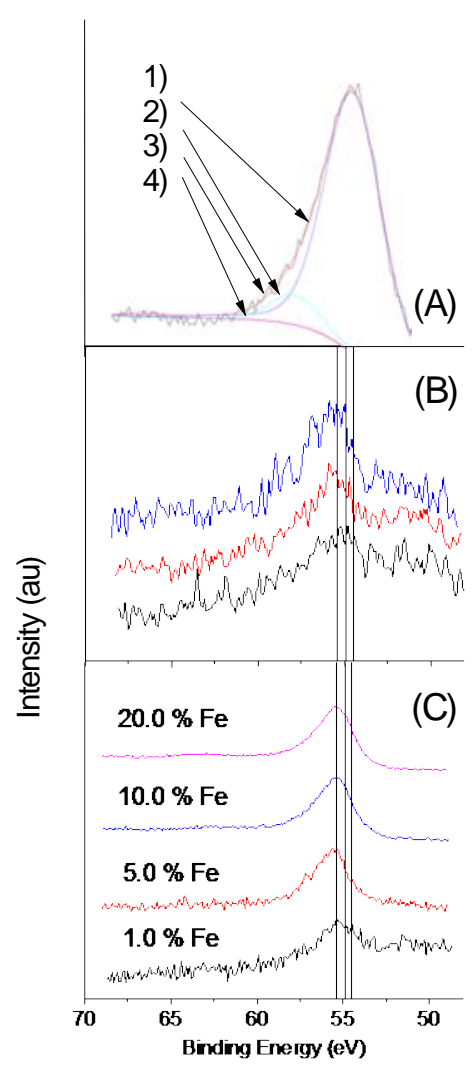

Figure 3 (color online) A) Curve Fit for Fe 3p peak in 10\% Fe doped Ce-Cl sample. Curve 1 is the fit for $\mathrm{Fe}^{3+}$ in $\mathrm{Fe}_{2} \mathrm{O}_{3}$ and curve 2 is for $\mathrm{Fe}^{3+}$ in $\mathrm{FeOOH}$. Peak position for $\mathrm{Fe}_{2} \mathrm{O}_{3}$ is $55.4 \mathrm{eV}$ and $\mathrm{FeOOH}$ is $56.25 \mathrm{eV}$ (14). Present in the fit is a summation curve (3) and a background curve (4). B) Fe 3p peak positions from XPS analysis of Ce-AmNit samples. The three drop lines indicate the reported $\mathrm{Fe} 3 p$ peak positions of $\mathrm{Fe}^{3+}$ in $\mathrm{Fe}_{2} \mathrm{O}_{3}$ and two $\mathrm{Fe}^{2+}$ positions reported for $\mathrm{FeO}$ at 55.4, 54.9, and $54.5 \mathrm{eV}$ respectively(14). C) Fe 3p peaks for iron doped Ce-Cl samples with drop lines corresponding to the ones mentioned in $\mathrm{B}$. 

may be found at: DOI: $10.1063 / 1.3688933$.
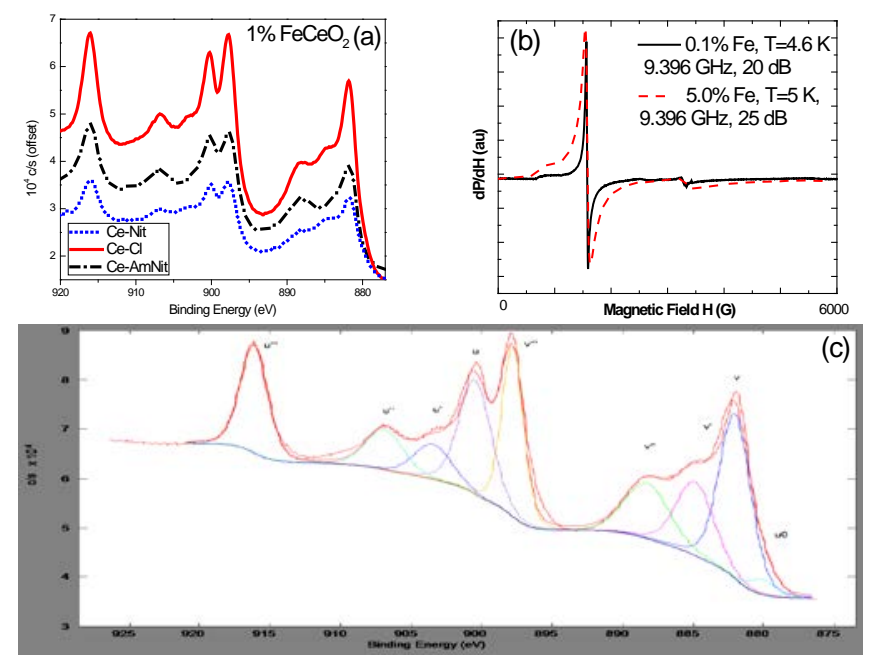

Figure 4 (color online) shows (a) XPS Ce3d spectral region for 3 1\%Fe-doped samples, (b) EPR spectra for $1 \%$ and $5 \%$ Fe-doped $\mathrm{Ce}-\mathrm{Cl}$ samples recorded at $\mathrm{T} \sim 5 \mathrm{~K}$ and (c) an example curve fit for determining $\mathrm{Ce}^{3+} / \mathrm{Ce}^{4+}$ ratio from XPS data . 

may be found at: DOI: $10.1063 / 1.3688933$.

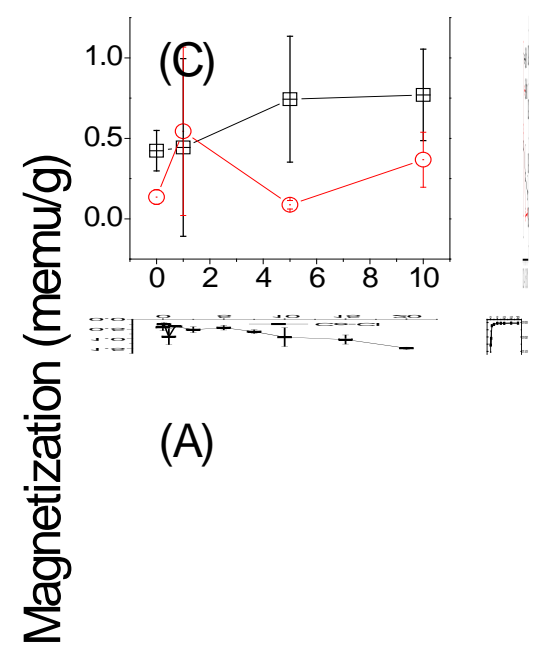

(D)

(B)

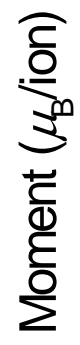

Fe $\%$

Figure 5 (color online) A-B) Magnetization and Moment of Ce-Cl samples. C-D) Magnetization and Moment of Ce-Nit and Ce-AmNit samples. 


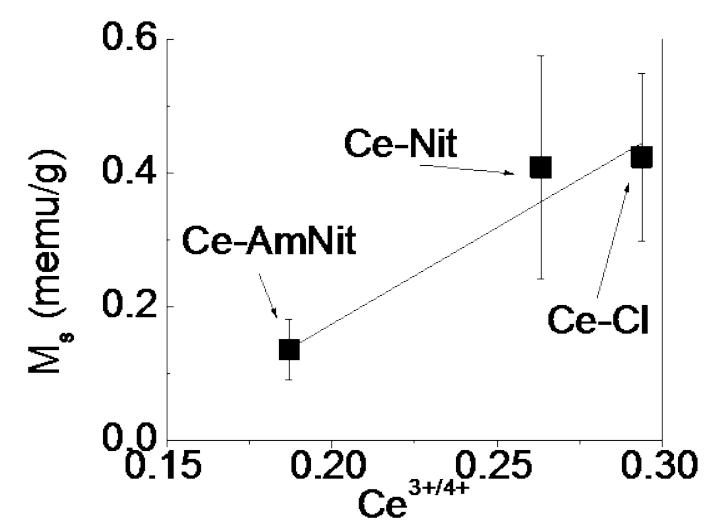

Figure 6 A) Average saturation magnetization $\mathrm{M}_{\mathrm{s}}$ of Fe doped ceria samples prepared using the three methods versus the $\mathrm{Ce}^{3+/ 4+}$ ratios, along with a linear fit shown for the three points. $\mathrm{Ce}^{3+/ 4+}$ ratios were determined from XPS analysis (15). 


\section{References:}

1) Sundaresan, A. et al, Physical Review B, 74, 161306, 2006

2) Sundaresan, A. et al, Nano Today, 4, 96-106, 2009

3) Ge M.Y. et al., Applied Physics Letters, 93. 062505, 2008

4) Li, Mingjie et al. IEEE Transactions on Magnetics, 44.11, 2708, Nov 2008

5) C. Binet et al, J. Phys. Chem., 98, 6392, 1997

6) Sharma, S.K. et al. Thin Solid Films. 519, 410-413, 2010

7) Dohčević-Mitrović, Z.D. et al. App. Phys. Letters. 96. 203104, 2010

8) Shah, Lubna R et al. Journal of Physics: Condensed Matter. 21. 486004, 2009

9) Vodungbo, G. et al. Appl Phys Lett., 90, 062510, 2007

10) Thurber, A. et al. Journal of Applied Phys., 101. 09N506, 2007

11) Misra, S. K. Journal of Applied Physics. 103. 07D122, 2008

12) Thurber, A. et al. Physical Review B. 76. 165206. 2007

13) Tiwari, A. et al. Appl Phys Lett. 88. 142511, 2006

14) D. Brion, Applications of Surface Science, Vol 5, 133-152, 1980

15) D. Karmakar et al, Phys. Rev. B 75, 144404 (2007) 